\title{
Prevenção secundária farmacológica de doenças cardiovasculares em população rural assistida por unidades básicas de saúde do município de Embu- Guaçu/SP
}

\author{
Reality of secondary prevention pharmacological therapy of cardiovascular diseases in the \\ city of Embu-Guaçu/SP
}

Fernanda Galvão Canda Kimura Dias ${ }^{1}$

Orcid: https://orcid.org/0000-0003-1423-5487

Carolina Nunes França ${ }^{3}$

Orcid: https://orcid.org/0000-0002-4167-4293

\author{
Yára Juliano ${ }^{2}$ \\ Orcid: https://orcid.org/0000-0002-8391-075X \\ Patrícia Colombo de Souza ${ }^{4}$ \\ Orcid: https://orcid.org/0000-0003-0247-4245
}

\begin{abstract}
Resumo
Introdução: As doenças cardiovasculares (DCV) constituem a primeira causa de morte no mundo. Em 2013, o Estudo da Carga Global das DCV estimou que 30\% de todas as mortes em todo o mundo foram causadas por DCV, sendo $28,7 \%$ dos óbitos em países em desenvolvimento e $26,6 \%$ em países desenvolvidos, de acordo com a Organização Mundial de saúde (OMS). Medidas preventivas, incluindo farmacoterapia, são importantes não apenas para diminuir a morbidade e mortalidade das DCV, mas também porque têm impacto na qualidade de vida. Segundo diretrizes internacionais de prevenção secundária das DCV recomenda-se tratamento medicamentoso ao longo da vida com antiagregante plaquetários (aspirina), betabloqueadores, estatinas e inibidores da enzima conversora de angiotensina (IECA). Objetivo: avaliar a prevenção secundária medicamentosa em pacientes que já apresentaram eventos cardiovasculares, frequentadores das Unidades de saúde da família do município de EmbuGuaçu. Método: estudo observacional, transversal e analítico. Participaram do estudo 67 pacientes que apresentaram pelo menos um evento cardiovascular e que responderam a um questionário contemplando dados sociodemográficos, identificação do evento cardiovascular, medicações prescritas, prescritores e o local de acompanhamento. Resultados: A média de idade dos pacientes foi de 64 anos. O AVE foi o evento mais prevalente $(p<0,0001)$ seguido de IAM. Não houve diferença significante na distribuição dos eventos segundo o sexo. Apenas $21 \%$ das prescrições estavam adequadas e em sua grande maioria, são acompanhados pelo cardiologista $(\mathrm{p}<0,0001)$. Dos pacientes acompanhados pela atenção primária à saúde, apenas $26 \%$ estavam com a prescrição adequada. Conclusão: A prevenção secundária medicamentosa dos pacientes estudados nessas UBS no município de Embu-Guaçu está longe de ser considerada ideal, pois apenas $26 \%$ dessas prescrições encontram-se adequadas segundo as diretrizes sobre prevenção secundária medicamentosa das DCV, e aquém de alguns estudos que chegam a ser $90 \%$.
\end{abstract}

Palavras-chave: fármacos cardiovasculares; doenças cardiovasculares; prevenção secundária

\begin{abstract}
\end{abstract}
Introduction: Cardiovascular diseases are the leading cause of death in the world. It encompasses Cardiovascular Diseases (CVD) Ischemic Heart Disease (ICD) with the largest representative being the entity: Acute Myocardial Infarction (AMI) and Cerebrovasculares Diseases (CBVD). In 2013, the Global Cargo Study (CSD) estimated that $30 \%$ of all death world were caused by CVD, with $28.7 \%$ of deaths in developing countries and $26.6 \%$ in developed countries, according to the World Health Organization

\footnotetext{
${ }^{1}$ E-mail: fernandakimura@yahoo.com.br

${ }^{2}$ E-mail: yjuliano@prof.unisa.br

${ }^{3}$ E-mail: carolufscar24@gmail.com

${ }^{4}$ E-mail: colombo@greco.com.br
} 
(WHO). Preventive measures, including pharmacotherapy, are important not only to decrease CVD morbidity and mortality but also because it has an impact on quality of life. According to international guidelines for secondary prevention of CVD, it is recommended to use lifelong medical treatment with antiplatelet agents (aspirin), beta blockers, statins and angiotensin converting enzyme inhibitors (ACEI). With increasing use of this treatment for secondary prevention, more than 104 lives could be saved and 191 recurrent ischemic events could be avoided with every 10,000 presentations. Objective: this research aimed to evaluate secondary drug prevention in patients who have already presented cardiovascular events, members of the family health units of the municipality of Embu-Guaçu. Method: A crosssectional observational and analytical study was conducted. The study included 67 patients who had at least one cardiovascular event, which answered a questionnaire that included socio-demographic data, identification of the cardiovascular event, and of the prescribed medications, prescribers and follow-up of these patients. Results: The mean age of patients was 64. The stroke was the most prevalent event ( $p$ $=0.0000$ ) followed by AMI. There wasn't significant difference in the distribution of events according to sex. Only $21 \%$ of prescriptions were adequate. Patients are mostly accompanied by cardiologists $(\mathrm{p}=$ $0.0000)$. Of patients treated by primary health care, only $26 \%$ had the proper prescription. Conclusion: Secondary drug prevention for patients studied in these UBS in the municipality of Embu-Guaçu is far from being considered ideal, as only $26 \%$ of these prescriptions are adequate according to the guidelines on secondary drug prevention for CVD, and below some studies that reach be $90 \%$.

Keywords: cardiovascular agentes; cardiovascular diseases; secondary prevention

\section{Introdução}

As doenças cardiovasculares (DCV) constituem a primeira causa de morte no mundo ${ }^{1-4}$. Elas são responsáveis por cerca de $20 \%$ de todas as mortes em indivíduos acima de $30 \operatorname{anos}^{4}$. A prevenção das doenças cardiovasculares pode ser primária, envolvendo a abordagem dos fatores de risco existentes ou não, em pessoas sem a doença cardiovascular como o sedentarismo, níveis de pressão arterial, colesterol, tabagismo, diabetes mellitus, entre outros. ${ }^{5}$ De acordo com a Organização Mundial da Saúde (OMS), a redução efetiva da mortalidade cardiovascular deve ser baseada em três pontos: 1-vigilância (mapeamento e monitorização epidemiológica das DCV), 2- prevenção (redução da exposição aos fatores de risco), 3- gestão (cuidados de saúde equitativos à pessoas com DCV). ${ }^{6}$ A identificação dos fatores de risco de maior prevalência populacional permitiu que programas de prevenção cardiovascular bem conduzidos em vários países a exemplo dos Estados Unidos, Canadá, Finlândia, Reino Unido, Austrália e Japão, conseguissem reduzir de forma expressiva a mortalidade por doenças cardiovasculares. $^{7}$
A prevenção secundária das DCV tem como objetivo reduzir o risco de um novo evento cardiovascular e morte, visando melhorar a sobrevida dos pacientes que sofreram o evento cardiovascular, por ser tratar de pacientes de muito alto risco. ${ }^{8,9}$ Medidas preventivas, incluindo farmacoterapia, são importantes não apenas para diminuir a morbidade e mortalidade das DCV, mas também porque tem impacto na qualidade de vida. ${ }^{10,11}$ Segundo diretrizes internacionais de prevenção secundária das DCV, recomenda-se tratamento medicamentoso ao longo da vida com antiagregantes plaquetários, betabloqueadores, estatinas e inibidores da enzima conversora de angiotensina (IECA). ${ }^{11-14}$

Com o aumento do uso desse tratamento para prevenção secundária, a cada 10.000 pacientes, mais de 104 vidas poderiam ser salvas e 191 eventos isquêmicos recorrentes poderiam ser evitados se a maioria desses pacientes com doenças crônicas fossem tratados na Atenção Primária. A dinâmica proposta pela Estratégia Saúde da Família (ESF), centrada na promoção da qualidade de vida e intervenção dos fatores que a colocam em risco, permite a identificação mais acurada 
e um melhor acompanhamento dos indivíduos com vistas à prevenção de doenças, principalmente as cardiovasculares, tendo um impacto sobre as taxas de incidência das mesmas. ${ }^{9}$

Diante do impacto causado pelas DCV na saúde do mundo e sendo a primeira causa de mortalidade na região estudada, pretende-se com este estudo, avaliar a prevenção secundária medicamentosa de pacientes que já apresentaram eventos cardiovasculares, frequentadores das Unidades Básicas de Saúde, com estratégia Saúde da Família do município de EmbuGuaçu/SP.

\section{Casuística e métodos}

Estudo observacional, transversal e analítico. Foram estudados todos os indivíduos que já sofreram evento cardiovascular, cadastrados nas seguintes Unidades Básicas de Saúde com Estratégia Saúde da Família no município de EmbuGuaçu, estado de São Paulo: UBS/ESF Sapateiro, UBS/ESF Recanto Lagoa Grande e UBS/ESF Penteado. A coleta ocorreu entre os meses de setembro a dezembro de 2016.

Embu-Guaçu situa-se na região metropolitana de São Paulo, sul da capital paulista. Faz divisa com o bairro de Parelheiros. No censo realizado em 2010 pelo IBGE, constatou-se uma população de 62.769 habitantes. ${ }^{15}$ Grande parte do seu território é área rural. O município conta atualmente com 17 Equipes Saúde da Família (ESF), alocadas nas UBS/ESF, apresentando uma cobertura de $90 \%$ da população com essa estratégia. As três equipes citadas foram escolhidas por terem características territoriais semelhantes estando todas em área rural. A população maior de 18 anos, assistida pelas 3 UBS/ESF giram em torno de 4.986 pessoas.

Por se tratar de um inquérito em que vários aspectos foram investigados de modo simultâneo, adotou-se para o cálculo amostral a proporção de evento de $50 \%$, com precisão de $5 \%$ e nível de $95 \%$ de confiança. Realizou-se ajuste para população finita e admitiram-se $20 \%$ de perda. A amostra calculada foi de 80 indivíduos, nas três UBS/ESF estudadas.

A seleção dos casos foi realizada junto com o líder comunitário, de modo que, chegar nos domicílios selecionados, estava de acordo com a disponibilidade do entrevistado. Nos domicílios visitados, todos os indivíduos que apresentavam casos de doença cardiovascular foram entrevistados e avaliados quanto ao uso da medicação específica para o evento. Foram incluídos adultos com idade maior ou igual a 18 anos e que aceitaram participar do estudo.

Como instrumento da pesquisa foi utilizado um questionário, desenvolvido pelo pesquisador, com dados sobre o tratamento medicamentoso necessário para a prevenção secundária das DCV. Foi avaliado se os medicamentos prescritos estavam de acordo com as diretrizes vigentes da Sociedade Brasileira de Cardiologia ${ }^{7}$ e conforme os estudos REACT e Euroaspire, ${ }^{16,17}$ conforme sua classe medicamentosa (antiagregantes plaquetários, betabloqueadores, inibidores da enzima conversora de angiotensina, bloqueadores do receptor de angiotensina e estatinas); foram verificados quais e quantos eventos cardiovasculares os pacientes estudados sofreram, tais como: Infarto Agudo do Miocárdio, hospitalização por angina, Acidente Vascular Encefálico, Cirurgia de revascularização, Doença arterial obstrutiva periférica; levantou-se também a procedência da primeira prescrição pós-evento (hospital, UBS, ambulatório de especialidades), e atualmente onde é realizado seu acompanhamento médico (UBS, hospital, ambulatório de especialidades); e por fim verificou-se o local onde os participantes retiram a medicação: Farmácia da UBS/ESF, Farmácia popular, Farmácia Comum.

O questionário foi aplicado pelos Agentes Comunitários de Saúde (ACS), que conhecem sua comunidade sabendo 
identificar e localizar quem apresentou DCV. A coleta foi realizada pelo ACS, individualmente, na residência do paciente em visitas domiciliares. Os ACS foram treinados previamente, pela pesquisadora, de maneira que houvesse uniformidade na coleta das informações.

O estudo foi submetido ao Comitê de Ética da Universidade Santo Amaro CAAE 58809916.2.0000.0081 e Número do Parecer: 1.695.918 e autorização da Secretaria Municipal de Saúde de EmbuGuaçu, com parecer favorável. Todos os participantes assinaram o Termo de Consentimento Livre e Esclarecido antes do início das entrevistas.

Foram descritos os medicamentos prescritos de acordo com o evento cardiovascular e mecanismo de ação. O Teste do Qui-quadrado ou Teste exato de Fisher foram utilizados para estudar possíveis associações entre variáveis estudadas, a análise de variância de Kruskal-Wallis para avaliar as idades entre diferentes eventos cardiovasculares e o teste
$\mathrm{G}$ de Cochran com a finalidade de estudar a concomitância dos eventos cardiovasculares tanto para mulheres, quanto para homens e para as USF. Foi utilizado o programa BioStat, versão $5.0 \mathrm{e}$ fixou-se em 0,05 ou $5 \%$ o nível de significância.

\section{Resultados}

Dentre os participantes do estudo, 68 apresentaram evento cardiovascular (Tabela 1). A população estudada foi acima de 18 anos e apresentou pelo menos um evento cardiovascular, correspondendo a $1,34 \%$ da população total. Houve uma distribuição homogênea entre mulheres e homens sem diferença significante quanto ao sexo (Tabela 2). A mediana das idades dos pacientes que participaram do estudo foi de 66,5, 63,5 e 66, nas Unidades do Penteado, Recanto e Sapateiro, respectivamente, e não houve diferença significante pelo teste de Kruskal-Wallis $(\mathrm{p}=0,3652)$.

Tabela 1 - Eventos cardiovasculares apresentados pelos pacientes incluídos no estudo

\begin{tabular}{cccc}
\hline IAM & Angina & AVC/AVE & DAOP \\
\hline $\mathrm{n}=24$ & $\mathrm{n}=15$ & $\mathrm{n}=27$ & $\mathrm{n}=2$ \\
$\%=28,2$ & $\%=17,6$ & $\%=31,7$ & $\%=2,3$ \\
\hline
\end{tabular}

AVC/AVE maior do que todos; $\mathrm{p}<0,0001$

IAM: Infarto Agudo do Miocárdio; AVC/AVE: Acidente Vascular Cerebral/Acidente Vascular Encefálico;

DAOP: Doença Arterial Obstrutiva Periférica

Tabela 2 - Presença de evento cardiovascular, segundo o sexo

\begin{tabular}{cccc}
\hline Evento Cardiovascular & $\begin{array}{c}\text { Feminino } \\
\text { \% Sim }\end{array}$ & $\begin{array}{c}\text { Masculino } \\
\text { \% Sim }\end{array}$ & $\begin{array}{c}\text { Teste do Qui-quadrado } \\
\text { Teste exato de Fisher }\end{array}$ \\
\hline IAM & 29 & 26,4 & $\mathrm{p}=0,89$ \\
Angina & 19,3 & 17 & $\mathrm{p}=0,94$ \\
AVC/AVE & 32,3 & 32,1 & $\mathrm{p}=0,93$ \\
DAOP & 0 & 3,8 & $\mathrm{p}=0,53$ \\
\hline
\end{tabular}

IAM: Infarto Agudo do Miocárdio; AVC/AVE: Acidente Vascular Cerebral/Acidente Vascular Encefálico; DAOP: Doença Arterial Obstrutiva Periférica

Os resultados referentes aos eventos cardiovasculares apresentados pelos pacientes estudados nas três unidades básicas de saúde da família mostraram que os AVE $(31 \%)$ predominam sobre os IAM $(28 \%)$, e ambos sobre a angina (17\%) e a doença obstrutiva crônica $(2,3 \%)$ (Tabela 1$)$.
Não houve diferenças significantes entre os eventos cardiovasculares avaliados nas mulheres e nos homens (Tabela 2).

Dos 68 pacientes avaliados, apenas $20,9 \%$ apresentaram prescrições medicamentosas contendo um representante de cada classe recomendada, considerada uma prescrição adequada. Não houve diferença significante entre as três unidades (Tabela 3). 
Tabela 3 - Pacientes com prescrições de pelo menos um medicamento recomendado de cada classe, segundo Unidade Básica de Saúde (UBS)

\begin{tabular}{|c|c|c|c|c|c|c|}
\hline \multirow{3}{*}{ UBS } & \multicolumn{4}{|c|}{ Prescrições adequadas } & \multicolumn{2}{|c|}{ Total de Sim } \\
\hline & \multicolumn{2}{|c|}{ Sim } & \multicolumn{2}{|c|}{ Não } & & \\
\hline & $\mathbf{N}$ & $\%$ & $\mathbf{N}$ & $\%$ & $\mathbf{N}$ & $\%$ \\
\hline Penteado & 6 & 42,8 & 20 & 29,8 & 26 & 23,1 \\
\hline Recanto & 3 & 21,5 & 17 & 25,3 & 20 & 15 \\
\hline Sapateiro & 5 & 35,7 & 16 & 23,9 & 21 & 23,8 \\
\hline Total & 14 & 100 & 53 & 100 & 67 & 20,9 \\
\hline
\end{tabular}

Teste exato de Fisher; $\mathrm{p}=0,74$

Conforme mostrado na Tabela 4, a maioria dos pacientes são acompanhados pelo cardiologista $(45,9 \%)$ e apenas $39 \%$ são acompanhados pela atenção primária (UBS/ESF). Desses pacientes acompanhados exclusivamente pela atenção primária, apenas 26,5\% apresentaram prescrição medicamentosa adequada (Tabela 5).

Tabela 4 - Especialidade médica do profissional que acompanha os pacientes estudados

\begin{tabular}{llll}
\hline UBS & CARDIOLOGIA & HOSPITAL & NEUROLOGIA \\
\hline $\mathrm{n}=34$ & $\mathrm{n}=40$ & $\mathrm{n}=06$ & $\mathrm{n}=07$ \\
$\%=39,0$ & $\%=45,9$ & $\%=6,9$ & $\%=8,0$ \\
\hline
\end{tabular}

CARDIOLOGISTA maior do que todos; $\mathrm{p}<0,0001$

Tabela 5 - Pacientes acompanhados exclusivamente no serviço de atenção primária, que apresentaram prescrições contendo um medicamento de cada classe de medicações recomendadas

\begin{tabular}{|c|c|c|c|c|c|c|}
\hline \multirow{3}{*}{$\begin{array}{l}\text { Unidade Básica } \\
\text { Saúde (UBS) }\end{array}$} & \multicolumn{4}{|c|}{ Prescrição Adequada } & \multirow{2}{*}{\multicolumn{2}{|c|}{ Total de Sim }} \\
\hline & \multicolumn{2}{|c|}{ Sim } & \multicolumn{2}{|c|}{ Não } & & \\
\hline & $\mathbf{N}$ & $\%$ & $\mathbf{N}$ & $\%$ & $\mathbf{N}$ & $\%$ \\
\hline Penteado & 4 & 44,4 & 10 & 40 & 14 & 28,5 \\
\hline Recanto & 2 & 22,2 & 6 & 24 & 8 & 25,0 \\
\hline Sapateiro & 3 & 33,4 & 9 & 36 & 12 & 25,0 \\
\hline Total & 9 & 100 & 25 & 100 & 34 & 26,5 \\
\hline
\end{tabular}

Teste do Qui-quadrado $\mathrm{X}^{2}=0,05 ; \mathrm{p}=0,97$

\section{Discussão}

As DCV são as principais causas de morte em quase todo o mundo, e a maioria dessas mortes são evitáveis com medidas preventivas, tanto na prevenção primária como na secundária ${ }^{1}$. Foi encontrado na população rural de Embu-Guaçu participante deste estudo que os AVE predominaram sobre os IAM, e ambos sobre todos os outros eventos, contradizendo alguns estudos que demonstram que a porcentagem de IAM se sobressai aos AVE, como o Epidemiological studies oh CHD and the evolution of preventive cardiology, o qual diz que doença cardíaca isquêmica é a manifestação predominante das $\mathrm{DCV}$, e causa $46 \%$ das mortes CV em homens e $38 \%$ em mulheres. A doença cerebrovascular é a forma de DCV com a segunda maior mortalidade - 34\% em homens e $37 \%$ nas mulheres ${ }^{18}$.

A ocorrência das DCV dá-se pela combinação de fatores de risco modificáveis (como tabagismo, alimentação, stress) e pelos não modificáveis como a idade, genética, gênero. Muitos estudos demonstram que a idade média do aparecimento das DCV em homens é de 55 anos e de 65 anos para as 
mulheres, sendo a idade média para ambos os gêneros em torno de $65 \operatorname{anos}^{17-19}$.

No presente estudo realizado na área rural no município de Embu-Guaçu, não houve diferenças na ocorrência de eventos cardiovasculares entre os sexos. Segundo dados da literatura, as mulheres com infarto agudo do miocárdio, além de serem cerca de dez anos mais velhas em relação aos homens, apresentam maior incidência de hipertensão arterial sistêmica, diabetes mellitus, coronárias normais e sinais clínicos de insuficiência cardíaca (apesar da fração de ejeção não ser menor que a do homem $)^{19}$. Ainda não está definido se a maior mortalidade em mulheres com infarto agudo do miocárdio ocorre pelo acometimento em idade mais avançada, pela diferença da incidência dos vários fatores de risco, ou se existe uma associação independente entre sexo feminino, morbidade e mortalidade pós-infarto agudo do miocárdio ${ }^{20}$.

Ainda considerando-se as diferenças entre os sexos, Bhatnagar e colaboradores ${ }^{21}$, em estudo epidemiológico realizado no reino Unido em 2014, mostraram que a prevalência de IAM em homens foi três vezes maior do que em mulheres; angina duas vezes mais prevalente em homens; AVE 2,53\% em homens e 1,99\% em mulheres.

$\mathrm{O}$ Epidemiological studies of $C H D$ and the evolution of preventive cardiology ${ }^{22}$ mostrou que doença cardíaca isquêmica é a manifestação predominante das DCV, e causa $46 \%$ das mortes $\mathrm{CV}$ em homens e $38 \%$ em mulheres. A doença cerebrovascular é a forma de DCV com a segunda maior mortalidade - 34\% em homens e $37 \%$ nas mulheres, contradizendo nosso estudo que encontrou que os AVE (31\%) predominaram sobre os IAM $(29 \%)$.

Atualmente, as diretrizes europeias de prevenção cardiovascular em pacientes com doença coronariana estabelecida, assim como a V Diretriz da Sociedade Brasileira de Cardiologia sobre Tratamento do Infarto Agudo do Miocárdio com Supradesnível do Segmento $\mathrm{ST}^{7}$, recomendam o uso de terapia antiplaquetária, agentes hipolipemiantes, um betabloqueador, e agentes de redução da PA adicionais no caso de uma PA sistólica acima de $140 \mathrm{mmHg}$ (preferencialmente os IECA e BRA) $)^{6,7}$. Os benefícios estabelecidos em prevenção secundária conferem indicação de uso do AAS em todos os pacientes com IAM com supra de ST por tempo indeterminado ${ }^{7}$. Em nosso estudo, apenas $64 \%$ dos pacientes que sofreram algum evento faziam uso de antiagregantes plaquetários.

Em relação aos betabloqueadores, a V Diretriz da Sociedade Brasileira de Cardiologia sobre Tratamento do Infarto Agudo do Miocárdio com Supradesnível do Segmento $\mathrm{ST}^{7}$ mostrou de forma inequívoca, ação dos bloqueadores betaadrenérgicos na redução dos eventos isquêmicos cardiovasculares, como a morte e reinfarto. Além disso, os Betabloqueadores devem ser usados de forma indefinida em todos os pacientes com IAM, exceto na presença de contraindicações absolutas ou relativas. Embora seja um medicamento com eficácia comprovada, e que impede a reincidência dos eventos, foi encontrado que apenas 59\% dos pacientes avaliados usavam betabloqueadores. Esse fato ocorre em todo o mundo, como mostrou o estudo europeu sobre prevenção secundária, por meio da intervenção para reduzir os eventos EUROASPIRE, que revelou que a prevenção das doenças cardiovasculares na prática clínica é inadequada nos países em que é necessário um gerenciamento mais efetivo em relação à prescrição medicamentosa ${ }^{17}$.

Em relação ao uso dos IECA e BRA, há argumentos para seu uso em todos os pacientes após IAM, em função da sua atuação antiisquêmica, antiaterosclerótica, e da consequente diminuição da recorrência de eventos isquêmicos, já evidenciados em vários estudos. De todas as classes medicamentosas em questão, a mais utilizada em nosso estudo foram os IECA e os BRA, utilizados por $88 \%$ dos pacientes 
sendo que $100 \%$ dos pacientes com angina fazem seu uso.

Os benefícios do uso de estatinas na prevenção secundária são comprovados e estudos clínicos randomizados também indicaram o benefício da utilização precoce e com estatina altamente potente ${ }^{7}$. Apesar desses benefícios comprovados pelas estatinas, somente $67 \%$ dos pacientes faziam uso no presente estudo. Dos pacientes que sofreram AVE, 67\% usavam as estatinas, e os infartados somente $61 \%$.

$\mathrm{O}$ estudo WHO PREMISSE ${ }^{23}$ descobriu que em países de baixa ou média renda econômica, menos de $40 \%$ dos pacientes com IAM receberam IECA e apenas $20 \%$ receberam estatinas. O estudo epidemiológico rural prospectivo urbano PURE $^{24}$ confirmou que a adesão com drogas para a prevenção secundária nos pacientes com DCV estabelecida era geralmente baixa e pior nos países de baixa renda: com mais de $80 \%$ não recebendo nenhum dos tratamentos necessários no sul da Ásia.

Dos 68 pacientes avaliados, apenas 20,9\% apresentaram prescrições medicamentosas contendo um representante de cada classe recomendada. Sabe-se que alguns podem ter contraindicações para algumas classes, porém a percentagem é muito aquém do esperado. Não houve diferença significante entre as três unidades, o que nos faz refletir sobre o serviço de atenção primária que deve coordenar o cuidado desses pacientes. Em estudo longitudinal realizado no Reino Unido, The UK National Health Sevice delivering equitable acroos the spectrum oh coronary disease $e^{25}$, em que foram analisadas prescrições de médicos da atenção primária para pacientes com IAM, entre 1999 a 2007, verificou-se que o uso de aspirina aumentou de $43 \%$ para $64 \%$, IECA e BRA de $22 \%$ para $59 \%$, os Betabloqueadores de $33 \%$ para $55 \%$ e estatinas de $38 \%$ para $72 \%$.

A atenção primária é o nível de saúde que além de ser a porta de entrada do SUS, é onde a integralidade, a longitudinalidade e a coordenação do cuidado devem ser diretrizes absolutas no cuidado dos pacientes. Em se tratando de doenças crônicas, essas diretrizes são irrevogáveis. No Brasil, com a estratégia saúde da família, o cuidado e acompanhamento dos pacientes com doenças crônicas não transmissíveis deveriam ser feitos $100 \%$ na atenção primária, mesmo com a necessidade de acompanhamento com nível secundário de atenção, a coordenação do cuidado é de responsabilidade da atenção primária.

Grande parte da responsabilidade pela prevenção secundária e gerenciamento de problemas efetivos em longo prazo associados à AVC são das equipes de atenção primária. Os médicos de família estão bem posicionados para implementar a prevenção secundária para esses pacientes. No entanto, as principais deficiências na distribuição da prevenção secundária após AVC foram vistas nas configurações de atenção primária.

Alguns estudos mostraram que a atenção primária é de extrema importância nos cuidados dos pacientes com DCV, tanto na prevenção primária quanto na secundária, e que após treinamento das equipes de saúde, houve melhora significante em relação à prevenção secundária, como apontaram os estudos CAGE e CHAMP $3,8,9,11,26,27$.

$\mathrm{O}$ presente estudo apresenta algumas limitações que devem ser levadas em consideração ao se analisar os resultados. Apesar de se tratar de uma amostra de base populacional, os indivíduos participantes foram limitados àqueles atendidos em apenas três das 17 equipes da Estratégia de Saúde da Família existentes no município, o que pode afetar a generalização dos resultados, levando à necessidade de continuidade do estudo para ampliar a amostra e também a participação de indivíduos atendidos em outras unidades.

\section{Conclusão}


A prevenção secundária medicamentosa dos pacientes estudados nessas UBS no município de Embu-Guaçu está longe de ser considerada ideal, pois apenas $26 \%$ dessas prescrições encontramse adequadas segundo as diretrizes sobre prevenção secundária medicamentosa das $\mathrm{DCV}$, e aquém de alguns estudos que chegam a ser $90 \%$.

\begin{abstract}
Contradizendo o esperado, pois todos os pacientes deveriam ser acompanhados pela atenção primária à saúde, a maioria dos pacientes são acompanhados pelo cardiologista (49\%), seguido das UBS com 32\%. Além disso, treinamento, capacitação e educação permanente devem fazer parte do dia a dia dos profissionais de saúde.
\end{abstract}

\section{Referências}

1. Leong DP, Joseph PG, McKee M, Anand SS, Teo KK, Schwalm J-D, et al. Reducing the global burden of cardiovascular disease, part 2: Prevention and treatment of cardiovascular disease. Circulation 2017;121(6):695-710.

2. Souza RKT, Bortoletto MSS, Loch RM, González DA, Matsuo T, Cabrera MAS, et al. Prevalência de fatores de risco cardiovascular em pessoas com 40 anos ou mais de idade, em Cambé, Paraná (2011): estudo de base populacional. Epidemiol Serv Saúde. 2013; 22(3):43544.

3. Chen CXR, Chan SL, Law TC, Choi SK, Chan KH. Secondary prevention of stroke: na evidencebased clinical audit in the primary care. Hong Kong Med J. 2011;17:469-77.

4. Mansur AP, Favarato D. Mortalidade por doenças cardiovasculares no Brasil e na região metropolitana de São Paulo: atualização 2011. Arq Bras Cardiol. 2012; 99(2):755-61.

5. World Health Organization. (WHO). Global status report on noncommunicable diseases 2010: Description of the global burden of NCDs, their risk factors and determinants.[periódico na internet]. 2011Apr [ acesso em 2016 Apr 17].[aproximadamente 176 p.]. Disponível em: http://www.who.int/nmh/publications/ncd_report2010/en/

6. Bansilal S, Castellano JM, Fuster V. Gloval Burden of CVD: focus on secondary of cardiovascular disease. Int J Cardiol. 2015;201(1): S1-7.

7. Piegas, LS, Timerman, A, Feitosa, GS, Nicolau, JC, Mattos, LAP, Andrade, MD. V Diretriz da Sociedade Brasileira de Cardiologia sobre Tratamento do Infarto Agudo do Miocárdio com Supradesnível do Segmento ST. Arq Bras Cardiol. 2015;105(2, Suppl. 1),1-121.

8. Brotons C, Maiques A, Mostaza J, Pinto X, Vilaseca J. Control lipídico en prevención secundaria: estudio multicéntrico, observacional en atención primaria. Aten Prim. 2004;34(2):81-6.

9. Sicras-Mainar A, Velasco-Velasco S, González-Rojas GN; Clemente-Igeño C, Rodríguez-Cid JL. Influencia de la morbilidad, control metabólico y uso de recursos de los sujetos ensituación de riesgo cardiovascular en el ámbito de la atención primaria de salud. Aten Primaria. 2008;40(9):447-54.

10. Poredos P, Jezovnik MK. Do the effects of secondary prevention of cardiovascular events in PAD patients differ from other atherosclerotic disease. Int J Mol Sci. 2015;16:14477-89.

11. Brasil CKOI, Avezum Jr A, Uint L, Del Monaco MI, Barros VM, Campos SYR, et al. Cardiovascular prevencion in coronary heart disease patients: guidelines implementacion in clinical practice. Rev Bras Cir Cardiovasc. 2013;28(2):238-47.

12. Therapeutic guidelines cardiovascular, 6th edn. Melbourne, Vic: Cardiovascular Expert Group. Therapeutic Guidelines, 2012.

13. Delsart P, Lemesle G, Lamblin N, Tricot O, Meurice T, Mycinski C, et al. Secondary medical prevention and clinical outcome in coronary artery disease patients with a history of noncoronary vascular intervention: a report from the CORONOR investigators. Eur J Prevent Cardiol. 2015;22(7):864-71.

14. Lieshout van J, Grol R, Campbell S, Falcoff H, Capell EF, Glehr M, et al. Cardiovascular risk management in patients with coronary heart disease in primary care: variation across countries 
and practices. An observational study based on quality indicators. BMC Fam Pract. 2012;13:96103.

15. Instituto Brasileiro de Geografia e Estatística. Disponível em: http://cidades.ibge.gov.br/

16. Berwanger O, Mattos LAP, Martin JFV, Lopes RD, Figueiredo EL, Magnoni D, Precoma DB, Machado CA, Guimarães JI, Andrade JP. Prescrição de terapias baseadas em evidências para pacientes de alto risco cardiovascular: estudo REACT. Arq.Brasil. de Cardiologia. 2013;100(33):212-220.

17. Kotseva K, Wood D, De Bacquer D, De Backer G, Rydén L, Jennings C. EUROASPIRE IV: A European Society of Cardiology survey on the lifestyle, risk factor and therapeutic management of coronary patients from 24 European countries. Eur J Prev Cardiol. 2016;23(6):636-48.

18. Munoz MA, Subirana I, Ramos R, Franzi A, Vila J, Marrugat J. Med Clin (Barc). Eficacia de um programa intensivo de prevenciòn secundaria de cardiopatia isquêmica tras 5 anos de intervención. 2008;130(14):521-5.

19. Simão AF, Precoma DB, Andrade JP, Correa Filho H, Saraiva JFK, Oliveira GMM, et al. I Diretriz brasileira de prevenção cardiovascular. Arq Bras Cardiol. 2013; 101(6):2-50.

20. Sex differences in the presentation and perception of symptoms among young patients with Myocardial Infarction: Evidence from VIRGO Study (Variation in Recovery: Role of gender on outcomes of young AMI patients). Circulation 2018;137(8):781-790.

21. Bhatnagar P, Wickramasinghe K, Williams J, Rayner M, Townsend N. The epidemiology of cardiovascular disease in the UK 2014. Heart 2015;101:1182-89.

22. Wong ND. Epidemiological studies of CHD and the evolution of preventive cardiology. Nat Ver Cardiol. 2014;11:276-89.

23. Mendis S, Abegunde D, Yussuf S, Ebrahim S, Shaper G, Ghannem H, et al. WHO study on Prevention of Recurrences of Myocardial Infarction and Stroke (WHO PREMISSE). Bull World Health Organization. 2005;83(11):820-9.

24. Yssuf S, Islam S, Chow CK, Rangarajan S, Dagenais, Diaz R, et al. Use of Secundary Prevention Drugs for Cardiovascular Disease in the Community in High-income, Middle-income, and Lowincome Countries (the PURE Study): a propesctive epidemiological survey. Lancet 2011;378(9798):1231-43.

25. Hawkins NM, Scholes S, Bajekal M, Love H, O'Flaherty M, Raine R, Capewell S. The UK National health service: delivering equitable treatment across the spectrum of coronary disease. Circ Cardiovasc Qual Out. 2013;6:208-16.

26. Escolano ER, Beltran DO, Tortosa MG, Palaón NA, Fort AC, Pérez JN, et al. El estúdio PROPESE: resultados de um novo modelo oeganizativo em atención primaria para para pacientes com cardiopatia isquêmica crónica basado em uma intervención multifactorial. Aten Primaria. 2014;46(supl 3):10-5.

27. Fonarow GC, Gawlinski A, Moughrabi S, Tillisch JH. Improved treatmentof coronary heart disease by implementation of Cardiac Hospitalization Atherosclerosis Management Program (CHAMP). Am J Cardiol. 2001;87(7):819-22.

\section{Como citar este editorial:}

Dias FGCK, Juliano Y, França CN, Souza PC. Prevenção secundária farmacológica de doenças cardiovasculares em população rural assistida por unidades básicas de saúde do município de Embu-Guaçu/SP. Rev. Aten. Saúde. 2021; 19(67): 9-17. 